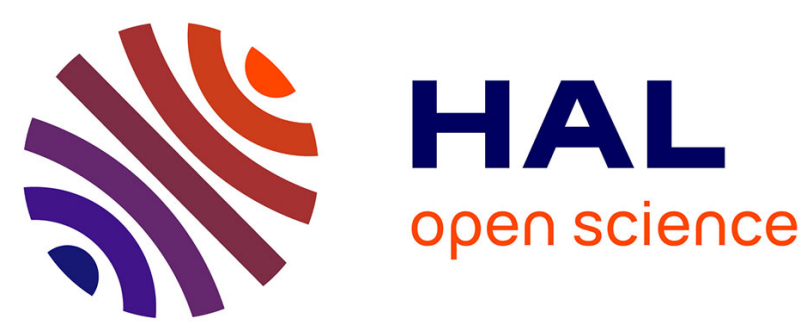

\title{
The OPERA experiment
}

C. Jollet

\section{To cite this version:}

C. Jollet. The OPERA experiment. $\mathrm{V}$ INTERNATIONAL CONFERENCE on NON-ACCELERATOR NEW PHYSICS, Jun 2005, Dubna, Russia. pp.1872-1875, 10.1134/S1063778806110093 . in2p3-00118917

\section{HAL Id: in2p3-00118917 https://hal.in2p3.fr/in2p3-00118917}

Submitted on 7 Dec 2006

HAL is a multi-disciplinary open access archive for the deposit and dissemination of scientific research documents, whether they are published or not. The documents may come from teaching and research institutions in France or abroad, or from public or private research centers.
L'archive ouverte pluridisciplinaire HAL, est destinée au dépôt et à la diffusion de documents scientifiques de niveau recherche, publiés ou non, émanant des établissements d'enseignement et de recherche français ou étrangers, des laboratoires publics ou privés. 


\author{
Cécile Jollet* \\ IReS, IN2P3-CNRS and University Louis Pasteur, Strasbourg, France
}

\begin{abstract}
The aim of the OPERA experiment is to provide an unambiguous evidence for the $\nu_{\mu} \leftrightarrow \nu_{\tau}$ oscillation by looking at the appearance of $\nu_{\tau}$ in a pure $\nu_{\mu}$ beam. This oscillation will be sought in the region of the oscillation parameters indicated by the atmospheric neutrino results. The experiment is part of the CNGS (Cern Neutrino beam to Gran Sasso) project. The $\nu_{\mu}$ beam produced at CERN will be sent towards the Gran Sasso underground laboratory, where the OPERA detector is under construction. The detector, the physics potential and performance for neutrino oscillation studies including the subleading $\nu_{\mu} \leftrightarrow \nu_{\mathrm{e}}$ search are presented.
\end{abstract}

PACS numbers:

\section{INTRODUCTION}

The "Appearance" long-baseline neutrino experiment OPERA [1] has been motivated by the atmospheric neutrino disappearance. Given the distance of $732 \mathrm{~km}$ between the neutrino source (at CERN) and the detectors (in the Gran Sasso underground laboratory), the CNGS beam (CERN Neutrino beam to Gran Sasso) [2] was designed in order to optimize the number of $\nu_{\tau}$ chargedcurrent (CC) interactions detectable at the Gran Sasso location. To be sensitive in the oscillation parameter region delimited mainly by the latest Super-Kamiokande results [3], the average energy of the CNGS beam is about $17 \mathrm{GeV}$. With the CERN SPS accelerator operating in a shared mode, $4.5 \times 10^{19}$ protons on target will be delivered per year. The number of $\nu_{\mu} \mathrm{CC}$ interactions is $2900 /(\mathrm{kt} \mathrm{yr})$. If the $\nu_{\mu} \leftrightarrow \nu_{\tau}$ oscillation hypothesis is confirmed, the number of $\tau$ 's produced via CC interaction at Gran Sasso will be of the order of 16/(kt yr) for $\Delta m^{2}=2.4 \times 10^{-3} \mathrm{eV}^{2}$ at full mixing.

\section{DETECTION TECHNIQUE}

The principle is to detect the $\tau$ leptons produced by $\nu_{\tau}$ interactions. According to the mean lifetime of the $\tau$ lepton, an unambiguous signature of its presence will be the detection of its decay topology. Consequently, the photographic emulsions will be used for the charged track detection with an accuracy better than $1 \mu \mathrm{m}$. This technique will allow one to localize the $\tau$-decay vertex ("kink"). Moreover, in order to increase the number of $\nu_{\tau}$ CC interactions, lead plates will be used for the target. The detection principle depicted in Fig. 1 takes into account these two conditions. The neutrinos interact with the 1-mm-thick lead target plates and the charged tracks are detected by the emulsion films. The kink between the $\tau$ track and the tracks produced by the particles following the $\tau$ decay makes an angle required to be greater than

\footnotetext{
*Electronic address: cecile.jollet@ires.in2p3.fr
}

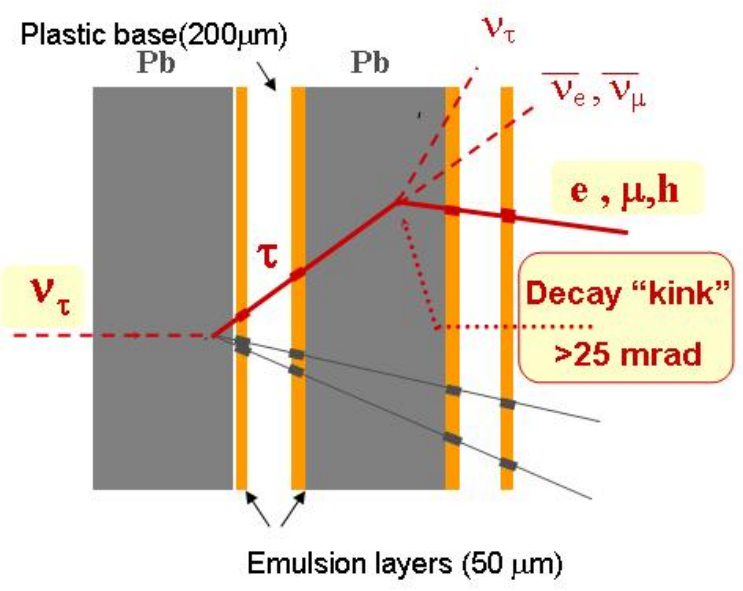

FIG. 1: $\nu_{\tau}$ and $\tau$ detection principle used by OPERA.

$25 \mathrm{mrad}$ to avoid background coming from multiple scattered tracks. Each track segment is reconstructed using 15 to 20 visible grains produced by the charged particles. The spatial resolution is of the order of $0.21 \mu \mathrm{m}$, while the angular resolution is about $2.1 \mathrm{mrad}$. On the reconstructed events, the kink search is performed, as well as the energy reconstruction of electromagnetic showers and the determination of momenta of charged particles by multiple scattering. All this information allows one to reconstruct the event kinematics.

\section{THE OPERA DETECTOR}

The detector is made of two identical supermodules, each one consisting of a target followed by a muon spectrometer. The schematic view is given in Fig. 2. The target combines "passive" elements such as emulsions with electronic detectors.

\section{A. Target bricks}

The base component of the detector is the target brick. Each brick is made of a sandwich of 56 1-mm-thick 


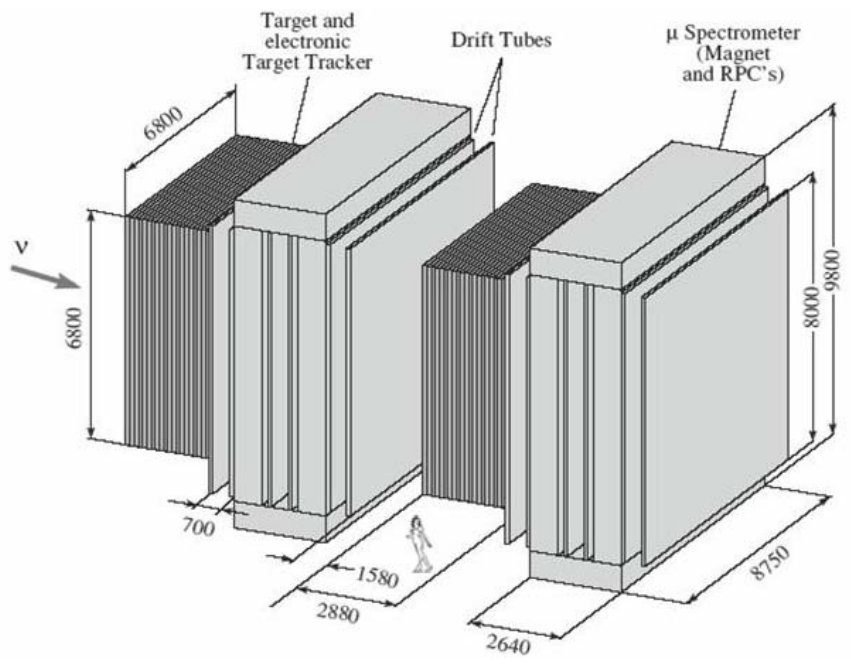

FIG. 2: Schematic view of the OPERA detector (the units are in $\mathrm{mm}$ ).

lead sheets interleaved with 56 emulsion layers $(50-\mu \mathrm{m}$ emulsion $+200-\mu \mathrm{m}$ plastic base $+50-\mu \mathrm{m}$ emulsion). The dimension of each brick is $12.8 \times 7.5 \times 10.3 \mathrm{~cm}^{3}$. The emulsion films are manufactured in Japan by FUJI company and the lead is a low-level radioactive one. The bricks are contained in light structures, $6.7 \mathrm{~m}$ high, of harmonic steel with horizontal trays. In order to reach a 1.8-kt target mass, 206336 bricks will be installed into 62 walls with 3328 bricks each. Each target is made of 31 brick walls and 31 associated electronic detector walls. The brick containing the interaction will be extracted, the emulsions developed and scanned with automatic microscopes having a scanning speed of $20 \mathrm{~cm}^{2} / \mathrm{h}$. The indication of the candidate brick to be extracted will be given by the electronic detector called target tracker.

\section{B. Target Tracker}

The main role of the target tracker is to localize the right brick to extract. Each wall of the target tracker will provide in $2 \mathrm{D}$ the position of the brick. It is composed of X and Y planes of 256 AMCRYS-H plastic scintillator strips $(6.8 \mathrm{~m} \times 2.6 \mathrm{~cm} \times 1 \mathrm{~cm})$. The scintillator strips are made of polystyrene with $2 \%$ p-terphenyl and $0.02 \%$ POPOP and are coated with a thin diffusing white layer of $\mathrm{TiO}_{2}$. The particle crossing the strips will create blue scintillation light. A wavelength-shifting fiber glued in a groove made in the center of the scintillator strip will absorb the blue light, reemit it at a green wavelength, and then propagate the light until the two extremities of the fiber (Fig. 3). Each target tracker wall is divided in four horizontal modules and four vertical modules. Each module is made of 64 scintillator strips, and the 64 fibers are connected to a multianode Hamamatsu photomultiplier tube at both strip ends. For a particle at the minimum

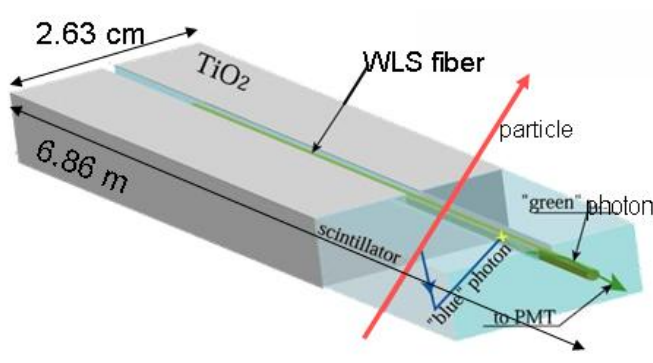

FIG. 3: Plastic scintillator strip and light detection technique used by the target tracker.

of ionization (2.15 MeV), at least five photoelectrons are detected by the photomultiplier. The target tracker having a detection efficiency of $99 \%$ will provide the trigger of the experiment. When the brick is found, a robot called BMS (Brick Manipulator System) will extract it. According to the trigger rate, around 40 bricks will be extracted per day. The Target Tracker will also help to reconstruct the event energy and initiate the muon tagging.

\section{Muon Spectrometer}

Its aim is to measure precisely the charge and the momentum of the muon and provide an efficient muon tagging. It is composed of two parts: an inner tracker and a precision tracker. The magnet of the inner tracker is made of two vertical walls having on top and bottom a flux return path surrounded by coils delivering a magnetic field of $1.55 \mathrm{~T}$. Each wall of the magnet is made of 12 iron slabs interleaved with RPCs (Resistive Plate Counters). The sampling structure of the RPCs is very similar to the one of the scintillator planes in the target. While the RPCs give the range of the muons, the precision tracker will measure precisely their momentum. It is made of drift tubes covering an area of $8 \times 8 \mathrm{~m}$. Drift tubes stations are placed in front of, in the middle of, and behind each dipole magnet. Figure 4 illustrates the spectrometer tracking strategy. The charge misidentification probability is around $0.1-0.3 \%$. The precision on the momentum measurement is lower than $20 \%$ for momentum lower than $50 \mathrm{GeV}$. Combining the muon spectrometer data with the target tracker information, the muon identification probability is greater than $95 \%$.

\section{Construction Status}

The detector is under construction in the Hall $\mathrm{C}$ of the Gran Sasso underground laboratory. Currently, the two magnets are installed and the first background data have been taken using RPCs. For the target, six empty brick walls and six target tracker walls are installed per month, which will permit having the first supermodule ready in 
